\title{
Pericortical Enhancement on Delayed Postgadolinium Fluid- Attenuated Inversion Recovery Images in Normal Aging, Mild Cognitive Impairment, and Alzheimer Disease
}

\author{
(D)W.M. Freeze, (DR.S. Schnerr, (D)W.M. Palm, DJ.F. Jansen, (DH.I. Jacobs, DE.I. Hoff, DF.R. Verhey, and (D)W.H. Backes
}

\begin{abstract}
BACKGROUND AND PURPOSE: Breakdown of BBB integrity occurs in dementia and may lead to neurodegeneration and cognitive decline. We assessed whether extravasation of gadolinium chelate could be visualized on delayed postcontrast FLAIR images in older individuals with and without cognitive impairment.
\end{abstract}

MATERIALS AND METHODS: Seventy-four individuals participated in this study (15 with Alzheimer disease, 33 with mild cognitive impairment, and 26 with normal cognition). We assessed the appearance of pericortical enhancement after contrast administration, MR imaging markers of cerebrovascular damage, and medial temporal lobe atrophy. Three participants who were positive for pericortical enhancement (1 with normal cognition and 2 with mild cognitive impairment) were followed up for approximately 2 years. In vitro experiments with a range of gadolinium concentrations served to elucidate the mechanisms underlying the postcontrast FLAIR signals.

RESULTS: Postcontrast pericortical enhancement was observed in 21 participants (28\%), including 6 individuals with Alzheimer disease (40\%), 10 with mild cognitive impairment (30\%), and 5 with normal cognition (19\%). Pericortical enhancement was positively associated with age $(P<.02)$ and ischemic stroke $(P<.05)$, but not with cognitive status $(P=.3)$. Foci with enhanced signal remained stable across time in all follow-up cases. The in vitro measurements confirmed that FLAIR imaging is highly sensitive for the detection of low gadolinium concentrations in CSF, but not in cerebral tissue.

CONCLUSIONS: Postcontrast pericortical enhancement on FLAIR images occurs in older individuals with normal cognition, mild cognitive impairment, and dementia. It may represent chronic focal superficial BBB leakage. Future longitudinal studies are needed to determine its clinical significance.

ABBREVIATIONS: $A D=$ Alzheimer disease; $A p o E=$ apolipoprotein $E ; M C I=$ mild cognitive impairment; $M M S E=$ Mini-Mental State Examination; $M T A=$ medial temporal lobe atrophy

$\mathbf{A}_{\mathrm{t}}^{\mathrm{l}}$ lzheimer disease $(\mathrm{AD})$ is the most common form of dementia, and is clinically characterized by progressive cognitive decline and associated functional impairment in daily living. Accumulating evidence suggests that microvascular dysregulation and BBB disruption are early events in the multifactorial disease cascade that leads to continuous neuronal loss. ${ }^{1,2}$ During the past decades, the development of gadolinium-based contrast agents

Received December 12, 2016; accepted after revision April 19, 2017.

From the Department of Psychiatry and Neuropsychology (W.M.F., H.I.., F.R.V.), Maastricht University, School for Mental Health and Neuroscience, Alzheimer Center Limburg, Maastricht, the Netherlands; Department of Radiology and Nuclear Medicine (W.M.F., R.S.S., J.F.J., W.M.P., W.H.B.), Maastricht University Medical Center, School for Mental Health and Neuroscience, Maastricht, the Netherlands; and Department of Neurology (E.I.H.), Zuyderland Medical Center Heerlen, Heerlen, the Netherlands.

This research was supported by Alzheimer Nederland (research grant WE-03-2012-40)

Paper previously presented in part at: International Congress on Vascular Dementia, October 16-18, 2015; Ljubljana, Slovenia; and Alzheimer's Association International Conference, July 22-28, 2016; Toronto, Ontario, Canada. has enabled detailed in vivo examination of BBB leakage by detection of extravasated gadolinium with postcontrast T1WI. Thus, previous studies reported subtle changes in vascular permeability in the cortex, white matter, and hippocampus in early-stage AD. ${ }^{3,4}$ Small changes in the dynamics of contrast agent leakage in ventricular CSF have also been reported in $\mathrm{AD} .{ }^{5}$ It is likely that vascular permeability changes also occur in vessels adjacent to the CSF along the cortical surface or leptomeninges. However, T1weighted BBB permeability imaging around the cortex in disease conditions with subtle changes to $\mathrm{BBB}$ integrity is probably difficult because the leptomeninges are highly vascularized and

Please address correspondence to Whitney M. Freeze, MSc, Department of Psychiatry and Neuropsychology, School for Mental Health and Neuroscience, Maastricht University, PO Box 616, 6200MD, Maastricht, the Netherlands; e-mail: w.freeze@maastrichtuniversity.nl

三 Indicates article with supplemental on-line table.

Indicates article with supplemental on-line photos.

http://dx.doi.org/10.3174/ajnr.A5273 
become relatively strongly enhanced due to high gadolinium concentrations within the blood vessels. Furthermore, when gadolinium chelate leaks into the CSF, convection and diffusion may dilute the contrast agent at concentrations that become too low to detect.

Delayed postcontrast FLAIR imaging yields up to 10 -fold higher sensitivity compared with postcontrast T1WI for the detection of low amounts of gadolinium chelate in the CSF. ${ }^{6,7}$ Previous studies that used postcontrast FLAIR imaging reported pericortical gadolinium leakage that appears as signal enhancement in the CSF in various clinical disease conditions with $\mathrm{BBB}$ leakage, including acute ischemic stroke and MS. ${ }^{8,9}$ The application of the postcontrast FLAIR technique has not yet been evaluated in patients in memory clinics and in normal aging, to our knowledge.

The primary objective of this study was to explore the utility of postcontrast FLAIR imaging in identifying pericortical enhancement in $\mathrm{AD}$, mild cognitive impairment (MCI), and age-matched cognitively healthy individuals. We hypothesized that pericortical $\mathrm{BBB}$ leakage is more common in patients with $\mathrm{AD}$ and MCI compared with cognitively healthy individuals and that it is associated with MR imaging markers of cerebrovascular damage. Finally, to investigate whether signal alterations could result from leakage of contrast medium into cortical tissue or into the CSF, we performed in vitro experiments to mimic the FLAIR signal effects of different concentrations of gadolinium in CSF and cerebral tissue.

\section{MATERIALS AND METHODS Participants}

Patients were prospectively recruited at 2 memory clinics (Maastricht University Medical Center and Zuyderland Medical Center Heerlen) between November 2014 and December 2016, and healthy controls were enrolled via a local newspaper advertisement in the same period. Detailed neuropsychological assessment, medical history, and educational level ${ }^{10}$ were recorded for all participants. Inclusion criteria for patients were a clinical diagnosis of subjective cognitive decline, ${ }^{11} \mathrm{MCI}^{12}$ or $\mathrm{AD},{ }^{13}$ and a Mini-Mental State Examination (MMSE) ${ }^{14}$ score of $\geq 20$. Control participants reported no subjective cognitive impairment and were screened to ensure cognitive health (MMSE score, $\geq 27$ ). Inclusion criteria for all participants were 55 years of age or older and eligibility for undergoing MR imaging and gadolinium contrast administration. Exclusion criteria for all participants included impaired renal function (estimated glomerular filtration rate of $<30 \mathrm{~mL} / \mathrm{min}$ ) and the presence of psychiatric or neurologic disorders that might have caused the cognitive impairment, other than $\mathrm{AD}$, cerebral small-vessel disease, or noninvalidating stroke. Participants with recent (eg, $<3$ months before inclusion) ischemic or hemorrhagic stroke were excluded. Informed consent was obtained from all participants before participation. This study was performed in accordance with the rules and regulations of the local institutional review boards and approved by the medical ethics committee in Maastricht.

\section{MR Imaging Protocol}

MR images were acquired on a 3T MR imaging system with a 32-channel head coil (Achieva TX; Philips Healthcare, Best, the
Netherlands). The imaging protocol was relatively long because other sequences that are not relevant to this article were included, and it was split into 2 separate sessions for the participants' comfort. During the first session without contrast administration, $3 \mathrm{D}$ T1-weighted gradient-echo (TR/TE, 8/4 ms; acquisition matrix, $256 \times 256$; 160 sections; $1-\mathrm{mm}$ section thickness) and 3D T2FLAIR (TR/TE/TI, 4800/290/1650 ms; acquisition matrix, $256 \times$ 256; 200 sections; 1 -mm section thickness) images were acquired, as well as multisection T2-weighted TSE and T2-weighted fastfield echo images with a section thickness of $5 \mathrm{~mm}$ in the transverse plane. During a second session (median time between scans 2 days; interquartile range, $0-5.5$ days), the same FLAIR sequence was acquired 16 minutes after intravenous administration of 0.1 $\mathrm{mmol} / \mathrm{kg}$ of gadobutrol in the antecubital vein (injection rate, 3 $\mathrm{mL} / \mathrm{s} ; 20-\mathrm{mL}$ saline flush).

\section{Image Analysis}

Pericortical enhancement was defined as hyperintense signal substantially brighter than the proximal parenchymal signal intensity, occurring in the subarachnoid space on postcontrast FLAIR images, but not on precontrast images. Two raters blinded to clinical data (W.M.F., W.M.P.) independently evaluated the scans for the presence of pericortical enhancement. Discrepancies and cases of uncertainty (Cohen $\kappa=0.50$ ) were evaluated during consensus meetings, after which both raters agreed on all cases. When present, pericortical enhancement was classified according to vascular territory (anterior, middle, or posterior cerebral artery), hemisphere (left/right), location (within a sulcus, along the cortical convexity, or within the longitudinal fissure), and number of foci.

White matter hyperintensities were scored on precontrast FLAIR images according to the Fazekas scale. ${ }^{15}$ Old lacunar infarcts were scored as subcortical round or ovoid structures of 3-15 $\mathrm{mm}$ in diameter with low signal intensity on FLAIR images and CSF-like signal intensity on T2WI. ${ }^{16}$ Cerebral microbleeds were scored as round or ovoid areas of signal void with associated blooming on T2-weighted fast-field echo images in cortical or subcortical regions, excluding potential mimics such as bilateral iron/calcium deposits in the basal ganglia, bone, or vessel flow voids. ${ }^{17}$ Old ischemic stroke of $>15 \mathrm{~mm}$ was scored on T2weighted TSE and FLAIR images, and old cerebral hemorrhage was scored on T2-weighted fast-field echo images. Medial temporal lobe atrophy (MTA) was rated bilaterally on coronal T1WI with a previously described visual rating scale. ${ }^{18}$ The average score of the left and right sides was taken as the final MTA score. Vascular markers and MTA were scored by an experienced neuroradiologist (W.M.P.), who was blinded to clinical data.

\section{Genetic Risk Factor Assessment}

Blood samples were collected and apolipoprotein E (ApoE) genotyping was performed with polymerase chain reaction. Individuals with at least $1 \varepsilon 4$ allele were classified as ApoE4-positive.

\section{Follow-Up}

One cognitively healthy participant and 2 patients with MCI who were positive for pericortical enhancement were followed up after 


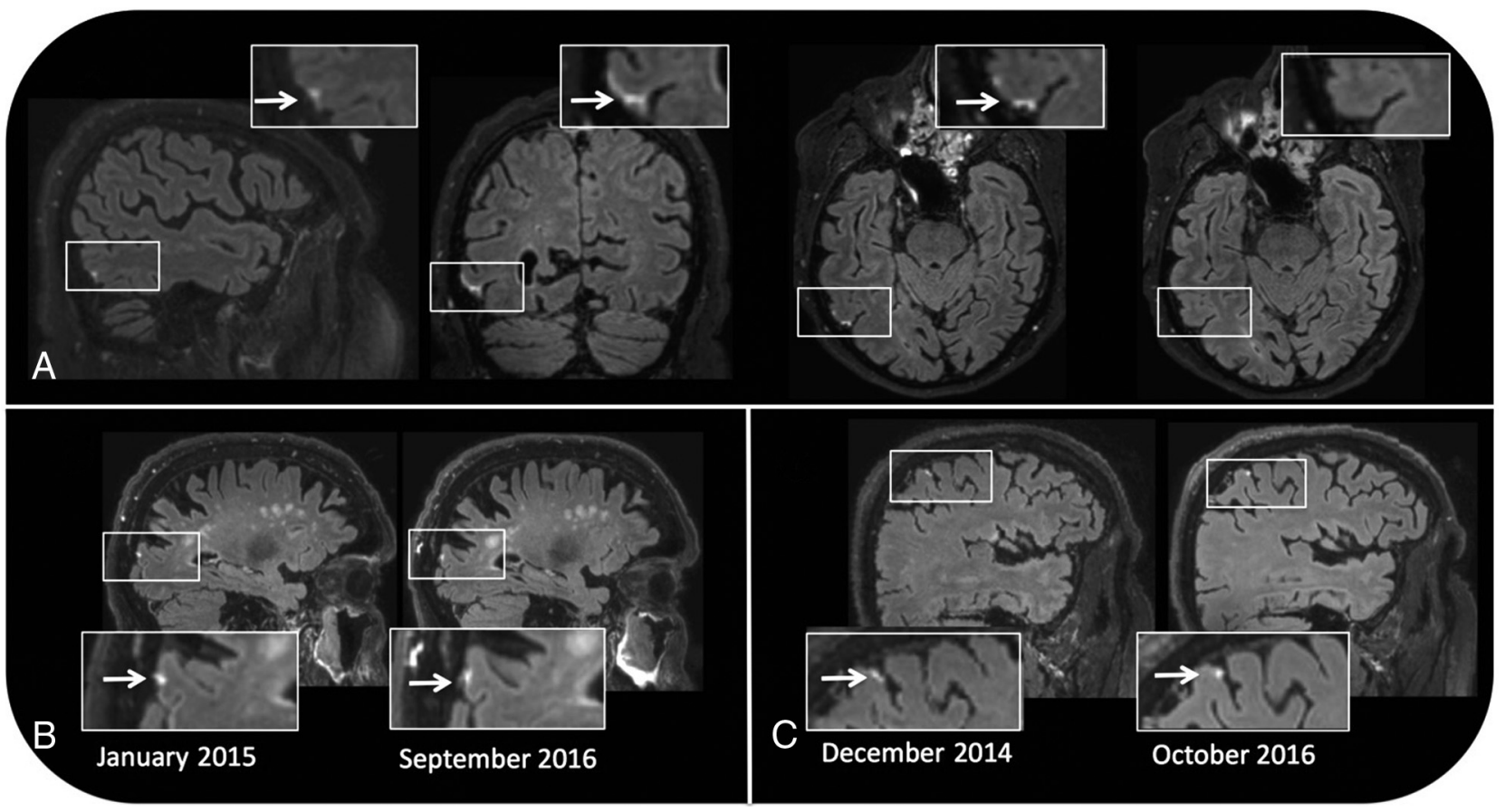

FIG 1. Pericortical enhancement is evident on sagittal, coronal, and transverse postcontrast FLAIR images of a 76-year-old male patient with MCI. No signal enhancement is apparent on the precontrast FLAIR image (upper right) (A). Pericortical enhancement remains stable across time in a male healthy control participant 87 years of age at baseline $(B)$ and in a male $\mathrm{MCl}$ participant 71 years of age at baseline $(C)$.

21-22 months. The same scan protocol was applied as described earlier, but all scans were acquired in 1 single session.

\section{Statistical Analyses}

Differences between individuals with and without pericortical enhancement were assessed with the $\chi^{2}$ or Fisher exact test (when applicable) for categoric variables. For continuous variables with a normal data distribution, independent 2 -sample $t$ tests were performed to compare the means of the 2 groups. Mann-Whitney $U$ tests were performed in case of non-normal data distribution.

To assess differences between the diagnostic groups (cognitively normal, MCI, and AD), we performed ANOVA for continuous variables with normal data distribution and Kruskal-Wallis tests for continuous variables with non-normal data distribution. The $\chi^{2}$ or Fisher exact test was used for categoric variables. A threshold of $\alpha<.05$ was used to determine statistical significance, all $P$ values were 2-tailed, and analyses were conducted with SPSS statistical software (Version 24.0; IBM, Armonk, New York).

\section{In Vitro Experiment}

Gadobutrol was dissolved in demineralized water in plastic Falcon tubes (50 mL; Corning, Corning, New York) in different concentrations $(0.004,0.008,0.016,0.031,0.063,0.125,0.250,0.500$, and $1.0 \mathrm{mmol} / \mathrm{L}$ ), mimicking in vivo gadolinium concentrations with a range of leakage severity in the CSF. The same gadolinium concentrations were adopted for a solution mimicking cerebral tissue, which was prepared by dissolving $0.08-\mathrm{mmol} / \mathrm{L} \mathrm{MnCl}_{2}$ in demineralized water. The $\mathrm{T} 1$ and $\mathrm{T} 2$ relaxation times of this $\mathrm{MnCl}_{2}$ solution were determined by using signal intensities from inversion recovery TSE images with $\mathrm{TR}=3000 \mathrm{~ms}$ and varying TIs $(50,100,200,400,600,800,1000$, and $1500 \mathrm{~ms})$ and T2- weighted images with a fixed TR $=453 \mathrm{~ms}$ and varying TEs (20, $40,60,80,100,120,140,160,180$, and $200 \mathrm{~ms})$. The T1 and T2 relaxation times (On-line Fig 1) of the tissue phantom approximated previously reported values of the cerebral cortex at $3 \mathrm{~T}$ with 1170 and 122 ms, respectively. ${ }^{19}$

The samples mimicking CSF and tissue with different concentrations of gadolinium were scanned with the T1 and FLAIR sequences described above.

\section{RESULTS}

\section{Neuroradiologic Imaging}

Seventy-four participants with a mean age of $71.2 \pm 6.2$ years were enrolled in this study, including 26 cognitively healthy participants (13 healthy controls and 13 with subjective cognitive decline), 33 with MCI, and 15 with AD. Participants' characteristics according to cognitive status (normal cognition, $\mathrm{MCI}$, or $\mathrm{AD}$ ) are provided in the On-line Table.

Twenty-one individuals showed evidence of abnormal pericortical enhancement on postcontrast FLAIR images (Fig 1). Overall, there were 32 foci, among which $10(31 \%)$ were located within the posterior, $9(28 \%)$ within the middle, and $13(41 \%)$ within the anterior cerebral artery territory. Fifteen foci $(47 \%)$ were located in the left and 17 (53\%), in the right hemisphere. Most foci were located on the cortical convexity $(12 / 32,38 \%)$ or within a sulcus $(12 / 32,38 \%)$, but $1(3 \%)$ traversed several sulci and $7(22 \%)$ were located within the longitudinal fissure. Pericortical enhancement was observed as a single focus in most cases $(13 / 21,62 \%)$. Three subjects with MCI and 2 subjects with dementia showed 2 foci, and 1 subject with MCI and 2 subjects with AD showed 3 foci (Fig 2).

In the 3 subjects positive for enhancement with a follow-up 


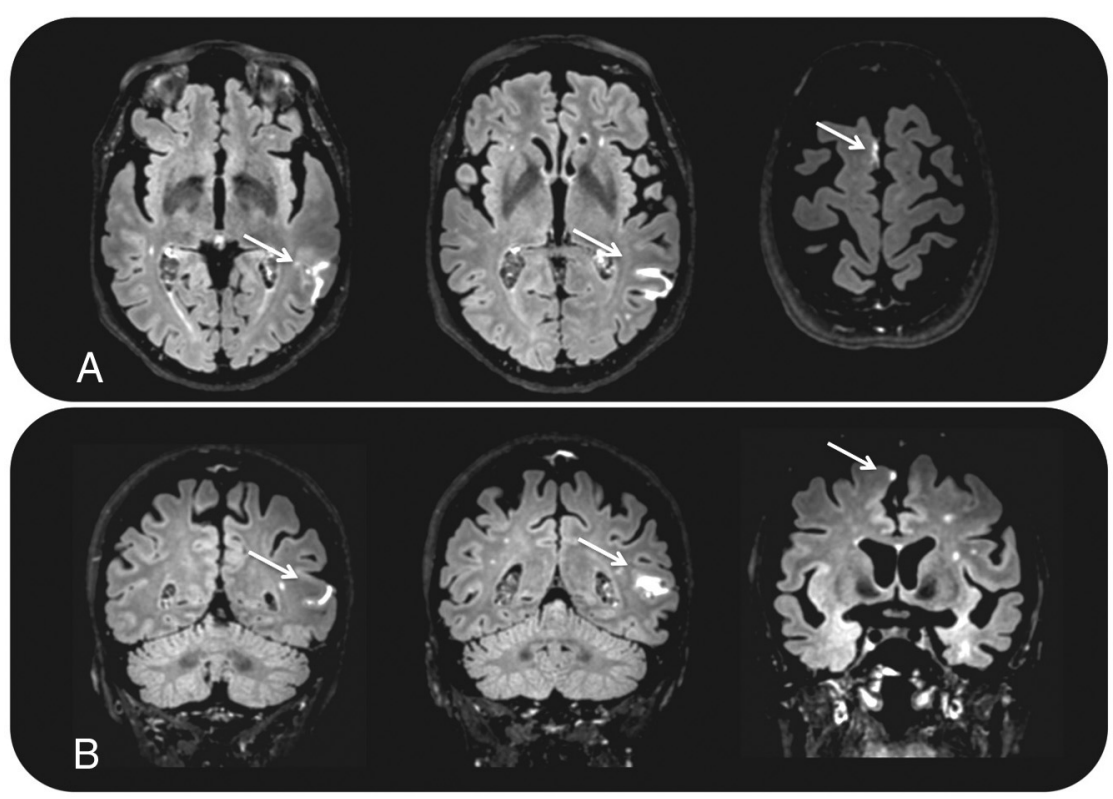

FIG 2. Two foci of pericortical enhancement on transverse $(A)$ and coronal $(B)$ postcontrast FLAIR images in a 73-year-old female participant with $A D$.

\section{Characteristics of pericortical enhancement}

\begin{tabular}{|c|c|c|c|}
\hline & \multicolumn{2}{|c|}{ Pericortical Enhancement } & \multirow[b]{2}{*}{$P$ Value } \\
\hline & Yes $(n=21)$ & No $(n=53)$ & \\
\hline \multicolumn{4}{|l|}{ Demographics } \\
\hline Age (mean) (SD) (yr) & $73.5(4.8)$ & $70.2(6.4)$ & $.02^{\mathrm{a}}$ \\
\hline Female sex (No) (\%) & $10(48)$ & $22(42)$ & .8 \\
\hline Clinical subtype (No) (\%) & & & .3 \\
\hline Normal cognition & $5(19)$ & $21(81)$ & \\
\hline $\mathrm{MCl}$ & $10(30)$ & $23(70)$ & \\
\hline$A D$ & $6(40)$ & $9(60)$ & \\
\hline \multicolumn{4}{|l|}{ MRI characteristics } \\
\hline Fazekas scale score (median) (IQR) & $1.0(1.0-3.0)$ & $1.0(1.0-2.0)$ & .4 \\
\hline Lacunar infarct present (No) (\%) & $7(33)$ & $8(15)$ & .11 \\
\hline Ischemic stroke present (No) (\%) & $4(19)$ & $3(6)$ & .18 \\
\hline Lacunar and/or ischemic stroke present (No) (\%) & $9(43)$ & $10(19)$ & $.04^{\mathrm{a}}$ \\
\hline Microbleed present (No) (\%) & $2(10)$ & $7(13)$ & 1.0 \\
\hline Hemorrhagic stroke present (No) (\%) & $1(5)^{\prime}$ & $1(2)^{\prime}$ & 1.0 \\
\hline MTA score (median) (IQR) & $1.0(0.5-2.25)$ & $0.5(0-1.5)$ & .08 \\
\hline Gadobutrol dosage (mean) (SD) (mL) & $7.3(1.2)$ & $7.3(1.1)$ & .98 \\
\hline \multicolumn{4}{|l|}{ Cognitive performance } \\
\hline MMSE (median) (IQR) ${ }^{\mathrm{b}}$ & $28(25-28)$ & $28(26-29)$ & .97 \\
\hline \multicolumn{4}{|l|}{ Genotype } \\
\hline ApoE4-positive (No) (\%) ${ }^{c}$ & $6(30)$ & $25(47)$ & .3 \\
\hline
\end{tabular}

Note:-IQR indicates interquartile range.

a Significant.

${ }^{b}$ Data were missing for 2 cases.

'Data were missing for 1 case.

time between 21 and 22 months, the foci remained stable across time regarding location, size, and shape (Fig 1).

In addition to the pericortical foci, 1 focus of signal enhancement was found in what appeared to be a perivascular space on the postcontrast but not on the precontrast FLAIR images (On-line Fig 2). No signal attenuation was observed in other fluid-containing structures, including lacunar infarcts, the ventricular system, or the eyes.

The group characteristics of individuals with pericortical enhancement are summarized in the Table. The prevalence per diagnostic group was 5/26 (19\%) in cognitively healthy individuals, $10 / 33(30 \%)$ in individuals with MCI, and 6/15 (40\%) in individ- uals with AD. Although the prevalence of pericortical enhancement was higher in the $\mathrm{AD}$ and MCI groups compared with the cognitively healthy individuals, there were no statistically significant group differences (Fisher exact test, $P=.3$ ). Individuals with pericortical enhancement were significantly older $(t[50]=2.4, P<.02)$ and more often had ischemic stroke $\left(\chi^{2}=4.5, d f=2\right.$, $P=.04)$ when lacunar infarct and ischemic stroke data were pooled. No associations were apparent among pericortical enhancement and sex, amount of contrast agent administered, MMSE score, ApoE genotype, and other MR imaging markers of cerebrovascular damage (Table). The location of pericortical enhancement did not correspond to the location of other MR imaging markers of cerebrovascular damage.

\section{In Vitro Measurements}

The phantom imaging experiment shows that relatively low concentrations of gadolinium in the CSF appear bright on FLAIR images, while at higher concentrations, the signal declines. On T1W1, low concentrations of gadolinium in the CSF appear dark, while at higher concentrations, the signal increases. In contrast, the FLAIR images of tissue phantoms with low gadolinium concentrations show only very little signal enhancement, while the signal decreases at higher concentrations. On T1WI, a gradual signal increase can be observed with increasing gadolinium concentrations (On-line Figs 3 and 4).

\section{DISCUSSION}

Delayed postcontrast FLAIR imaging revealed pericortical signal enhancement in $28 \%$ of all participants who underwent MR imaging. This signal enhancement likely results from leakage of gadolinium chelate through cortical or leptomeningeal vessels and, as such, represents a marker for focal loss of BBB integrity. Although pericortical enhancement occurred more often in MCI or AD compared with cognitively healthy individuals, there were no statistical group differences. Pericortical enhancement was associated with increasing age and ischemic stroke. Most interesting, in the 3 subjects who were followed up, the pericortical enhancement remained stable for 21-22 months. A possible explanation for this imaging phenomenon is chronic inflammation with BBB disruption in the leptomeninges or superficial cortical vessels. 
The potential of postcontrast FLAIR imaging for the detection of contrast agent leakage through the BBB was already described by Mathews et al in $1999 .{ }^{20}$ Since then, several studies on the application of this technique have been published. Many of these describe postischemic diffuse enhancement in the CSF in 1 or both hemispheres, a phenomenon called "hyperintense acute reperfusion marker." ${ }^{1,9,21,22}$ Similar to its acute presentation after ischemic stroke or transient ischemic attack, the appearance of the hyperintense acute reperfusion marker has also been described after hemorrhagic stroke, cardiac surgery, and carotid revascularization. ${ }^{23-25}$ These findings suggest a common process of rapid pericortical BBB leakage following cerebral ischemia or hypoxia. In addition to its relationship with ischemia, pericortical BBB leakage on postcontrast FLAIR images has been described in infectious diseases of the CNS, including meningitis and MS. ${ }^{8,26}$ Together, these studies suggest that (chronic) hypoxic and/or inflammatory processes in the cortex and leptomeninges may underlie this neuroimaging phenomenon. Pericortical enhancement is likely not specific for a particular disease and also occurs in the context of normal aging. ${ }^{27,28}$

We found no association between pericortical enhancement and diagnostic group, ApoE genotype, MTA score, or global cognition. Hence, the clinical relevance of pericortical enhancement in our study population remains unclear. Although delayed postcontrast FLAIR imaging is not routinely used in clinical examinations, the CSF signal change should not be confused with other causes of signal increase in the subarachnoid space on FLAIR images such as subarachnoid hemorrhage or meningeal metastasis. $^{29}$

Because the signal enhancement on postcontrast FLAIR images appears very close to the cortical surface, we aimed to assess whether the focal enhancement occurs in the CSF or cortical tissue. Our phantom study showed that FLAIR imaging is relatively insensitive for the detection of gadolinium in brain tissue, while strong signal enhancement is observed in CSF, mimicking phantom solutions at low gadolinium concentrations (ie, 10-500 $\mu \mathrm{mol} / \mathrm{L})$. We therefore interpret the pericortical enhancement as leakage and accumulation of low gadolinium concentrations in the pericortical CSF.

Previous studies have shown 2 advantages of postcontrast FLAIR imaging over T1WI in detecting pericortical BBB leakage. First, postcontrast FLAIR imaging is superior to postcontrast T1WI in detecting low gadolinium concentrations (ie, 10-500 $\mu \mathrm{mol} / \mathrm{L}$ ) in the CSF. ${ }^{7,20}$ Because of the long T1 relaxation time of CSF, FLAIR is relatively sensitive to subtle shortening of the T1 relaxation time caused by low concentrations of gadolinium. Second, previous work has shown that unlike T1WI, FLAIR is rather insensitive to the effects of contrast medium within blood vessels because the shortening of the T2 relaxation time at higher gadolinium concentrations and the effects of blood flow both result in a strong signal decay. ${ }^{26}$

The strengths of this study include the availability of both preand postcontrast FLAIR images obtained in individuals with a wide range of cognitive performance. The availability of other MR imaging sequences allowed us to look for associations between postcontrast FLAIR enhancement and various markers of cerebrovascular damage. The timeframe of 16 minutes between contrast injection and FLAIR imaging proved suitable for detecting pericortical enhancement in a relatively high number (ie, 28\%) of participants in our study.

Limitations of this study include the relatively small sample size, which limits our ability to draw conclusions about the presence or absence of associations between BBB leakage and variables of interest. Because we did not include markers more specific for neurodegeneration than MTA and $\mathrm{AD}$, the biomarkers amyloid $\beta$ and $\tau$ were not measured, and their association with pericortical enhancement remains unknown. Although the sensitivity of postcontrast FLAIR imaging compared with T1WI was assessed in our phantom study, we did not set out to make this comparison in vivo. We expect that the enhancement of the leptomeningeal vessels due to intravascular gadolinium complicates the detection of focal pericortical enhancement on postcontrast T1weighted images.

Future studies are needed to elucidate the pathophysiology and clinical relevance of pericortical BBB leakage in relation to neurodegeneration. The potential of pericortical enhancement as a biomarker for cognitive decline and dementia could be explored by future studies that compare the cognitive profile of individuals with and without pericortical enhancement within the same diagnostic group (ie, normal cognition, MCI, dementia) and by following individuals across time to assess conversion rates to MCI or dementia. The optimal timeframe between contrast injection and scan acquisition remains to be explored, as well as whether this phenomenon also occurs in younger individuals. In the future, larger study samples and longitudinal studies may shed light on the clinical consequences following signs of pericortical BBB disruption in aging, dementia, and other disorders of the CNS.

\section{CONCLUSIONS}

This study identified focal pericortical BBB leakage on delayed postcontrast FLAIR images in a substantial proportion of patients with AD and MCI as well as cognitively healthy participants. Pericortical signal enhancement was associated with increasing age and previous ischemic stroke. In all 3 follow-up cases, the focal BBB leakage persisted and remained stable across time. The results of our study demonstrate the suitability of postcontrast FLAIR imaging as a tool for detecting possible chronic pericortical vascular leaks in a memory clinic setting and in cognitively healthy older individuals.

\section{ACKNOWLEDGMENTS}

We thank Danique Hellebrekers and Tiny Simons for their assistance with participant inclusion; Jos Slenter, Ivo Pooters, and Joost Löring for their contribution to the phantom study; and Paul Hofman for his advice on image interpretation.

Disclosures: Whitney M. Freeze-RELATED: Grant: Alzheimer Nederland, Comments: This grant supports study costs including MRI scanning, analysis of blood samples, and my salary as a PhD student in the Netherlands*; Support for Travel to Meetings for the Study or Other Purposes: Alzheimer Nederland, Comments: I received $€ 500$ to support my attendance to present part of this work at the Alzheimer's Association International Conference that took place in Toronto, Ontario Canada in July 2016. Heidi I. Jacobs—RELATED: Grant: Alzheimer Nederland; Comments: money for MRI scans; UNRELATED: Employment: Netherlands Organisation for Scientific Research, Comments: grant for salary of Dr Heidi Jacobs*; Grants/ Grants Pending: Memorabel ZonMw, Comments: grant submitted on December 8*; Travel/Accommodations/Meeting Expenses Unrelated to Activities Listed: Alzheimer's Association, Human Amyloid Imaging, Alzheimer Nederland, Comments: travel fellowship for conference. * Frans R. Verhey-UNRELATED: Grant: ZonMw National 
Research Organisation*. Walter H. Backes—RELATED: Grant: Stichting Alzheimer Onderzoek Nederland. * ${ }^{*}$ Money paid to the institution.

\section{REFERENCES}

1. Zlokovic BV. Neurovascular pathways to neurodegeneration in Alzheimer's disease and other disorders. Nat Rev Neurosci 2011;12: 723-38 CrossRef Medline

2. Iturria-Medina Y, Sotero RC, Toussaint PJ, et al; Alzheimer's Disease Neuroimaging Initiative. Early role of vascular dysregulation on late-onset Alzheimer's disease based on multifactorial data-driven analysis. Nat Commun 2016;7:11934 CrossRef Medline

3. Montagne A, Barnes SR, Sweeney MD, et al. Blood-brain barrier breakdown in the aging human hippocampus. Neuron 2015;85: 296-302 CrossRef Medline

4. van de Haar HJ, Burgmans S, Jansen JF, et al. Blood-brain barrier leakage in patients with early Alzheimer disease. Radiology 2016; 281:527-35 CrossRef Medline

5. Starr JM, Farrall AJ, Armitage P, et al. Blood-brain barrier permeability in Alzheimer's disease: a case-control MRI study. Psychiatry Res 2009;171:232-41 CrossRef Medline

6. Mamourian AC, Hoopes PJ, Lewis LD. Visualization of intravenously administered contrast material in the CSF on fluid-attenuated inversion-recovery MR images: an in vitro and animal-model investigation. AJNR Am J Neuroradiol 2000;21:105-11 Medline

7. Köhrmann M, Struffert T, Frenzel T, et al. The hyperintense acute reperfusion marker on fluid-attenuated inversion recovery magnetic resonance imaging is caused by gadolinium in the cerebrospinal fluid. Stroke 2012;43:259-61 CrossRef Medline

8. Absinta M, Vuolo L, Rao A, et al. Gadolinium-based MRI characterization of leptomeningeal inflammation in multiple sclerosis. $\mathrm{Neu}$ rology 2015;85:18-28 CrossRef Medline

9. Latour LL, Kang DW, Ezzeddine MA, et al. Early blood-brain barrier disruption in human focal brain ischemia. Ann Neurol 2004;56: 468-77 CrossRef Medline

10. Verhage F. Intelligence and age in a Dutch sample. Human Development 1965;8:238-45 CrossRef

11. Jessen F, Amariglio RE, van Boxtel M, et al; Subjective Cognitive Decline Initiative (SCD-I) Working Group. A conceptual framework for research on subjective cognitive decline in preclinical Alzheimer's disease. Alzheimers Dement 2014;10:844-52 CrossRef Medline

12. Petersen RC. Mild cognitive impairment as a diagnostic entity. J Intern Med 2004;256:183-94 CrossRef Medline

13. McKhann G, Drachman D, Folstein M, et al. Clinical diagnosis of Alzheimer's disease: report of the NINCDS-ADRDA Work Group under the auspices of Department of Health and Human Services Task Force on Alzheimer's Disease. Neurology 1984;34:939-44 CrossRef Medline

14. Folstein MF, Folstein SE, McHugh PR. "Mini-Mental State": a practical method for grading the cognitive state of patients for the clinician. J Psychiatr Res 1975;12:189-98 CrossRef Medline

15. Fazekas F, Chawluk JB, Alavi A, et al. MR signal abnormalities at $\mathbf{1 . 5}$
T in Alzheimer's dementia and normal aging. AJR Am J Roentgenol 1987;149:351-56 CrossRef Medline

16. Wardlaw JM, Smith EE, Biessels GJ, et al; STandards for ReportIng Vascular changes on nEuroimaging (STRIVE v1). Neuroimaging standards for research into small vessel disease and its contribution to ageing and neurodegeneration. Lancet Neurol 2013;12:822-38 CrossRef Medline

17. Greenberg SM, Vernooij MW, Cordonnier C, et al; Microbleed Study Group. Cerebral microbleeds: a guide to detection and interpretation. Lancet Neurol 2009;8:165-74 CrossRef Medline

18. Scheltens P, Leys D, Barkhof F, et al. Atrophy of medial temporal lobes on MRI in "probable" Alzheimer's disease and normal ageing: diagnostic value and neuropsychological correlates. J Neurol Neurosurg Psychiatry 1992;55:967-72 CrossRef Medline

19. Wansapura JP, Holland SK, Dunn RS, et al. NMR relaxation times in the human brain at 3.0 Tesla. J Magn Reson Imaging 1999;9:531-38 CrossRef Medline

20. Mathews VP, Caldemeyer KS, Lowe MJ, et al. Brain: gadoliniumenhanced fast fluid-attenuated inversion-recovery MR imaging. Radiology 1999;211:257-63 CrossRef Medline

21. Warach S, Latour LL. Evidence of reperfusion injury, exacerbated by thrombolytic therapy, in human focal brain ischemia using a novel imaging marker of early blood-brain barrier disruption. Stroke 2004;35:2659-61 CrossRef Medline

22. Förster A, Wenz H, Groden C. Hyperintense acute reperfusion marker on FLAIR in a patient with transient ischemic attack. Case Rep Radiol 2016;2016:9829823 CrossRef Medline

23. Kidwell CS, Burgess R, Menon R, et al. Hyperacute injury marker (HARM) in primary hemorrhage: a distinct form of CNS barrier disruption. Neurology 2011;77:1725-28 CrossRef Medline

24. Merino JG, Latour LL, Tso A, et al. Blood-brain barrier disruption after cardiac surgery. AJNR Am J Neuroradiol 2013;34:518-23 CrossRef Medline

25. Cho AH, Suh DC, Kim GE, et al. MRI evidence of reperfusion injury associated with neurological deficits after carotid revascularization procedures. Eur J Neurol 2009;16:1066-69 CrossRef Medline

26. Fukuoka H, Hirai T, Okuda T, et al. Comparison of the added value of contrast-enhanced 3D fluid-attenuated inversion recovery and magnetization-prepared rapid acquisition of gradient echo sequences in relation to conventional postcontrast T1-weighted images for the evaluation of leptomeningeal diseases at 3T. AJNR Am J Neuroradiol 2010;31:868-73 CrossRef Medline

27. Erdő F, Denes L, de Lange E. Age-associated physiological and pathological changes at the blood-brain barrier: a review. J Cereb Blood Flow Metab 2017;37:4-24 CrossRef Medline

28. Farrall AJ, Wardlaw JM. Blood-brain barrier: ageing and microvascular disease-systematic review and meta-analysis. Neurobiol Aging 2009;30:337-52 CrossRef Medline

29. Stuckey SL, Goh TD, Heffernan T, et al. Hyperintensity in the subarachnoid space on FLAIR MRI. AJR Am J Roentgenol 2007;189: 913-21 CrossRef Medline 